\title{
Analysis of Stock Market Prediction Models Using Deep Learning
}

\author{
Harmanjeet Singh and Anand Kr. Shukla \\ University Institute of Computing, Chandigarh University, SAS Nagar, Mohali, India
}

\section{ABSTRACT}

In era of Internet and Big Data where investors are open to share sentiments or opinion about share price for discussion which leads to generate massive amount of unstructured data. This unstructured data further affects the direction of stock price on the basis of investor's emotions and sentiments. In recent years, Deep Learning techniques are extensively explored to predict stock fluctuation using historical data, technical indicators and sentiment analysis. This paper inspects the recent literature in the area of Deep Learning Neural Network, Sentiment Analysis, Data Mining, Fuzzy logic and Machine Learning systems to predict the stock market movement. Due to its nonlinear approach, stock market prediction cannot be relying on traditional methods including fundament analysis and technical analysis. In this paper, we give close eye on summarization enhancements, challenges, future scope and categorized research papers published in this field since 2011. Many recent proposed algorithm's methodologies are studied and presented briefly in this paper. Although discussion was based on predictor techniques, trading strategies and evaluation matrices.

\section{KEY WORDS: STOCK MARKET, SENTIMENT ANALYSIS, DEEP LEARNING, MACHINE LEARNING, DATA MINING.}

\section{INTRODUCTION}

STOCK prices forecasting is widely studied topic in various research fields including finance, trading, economy and of course computer science. The core study generally focusses on forecast the direction of stock's future prices on basis of stock could be buy or sell in profitable position. To make investment decision, most professional trader's studies fundamental or/and technical analysis of the stock prices movement in future. Fundamental Analysis is a traditional way to determine the intrinsic value of company's stock which all depends upon company's revenues, competitors, balance sheet, cash flow where as Technical Analysis is solely based upon study of stock's historical price pattern and Volume Information. Most technical professionals forecast future price movement of stock with support of analyze price chart of stock to determine price pattern. Moreover, stock market plays a critical role in any country's economic growth. Due

Biosc Biotech Res Comm P-ISSN: 0974-6455 E-ISSN: 2321-4007

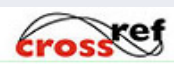

Identifiers and Pagination

Year: 2021 Vol: 14 No (9) Special Issue

Pages: $74-80$

This is an open access article under Creative

Commons License Attribn 4.0 Intl (CC-BY).

DOI: $h t t p: / / d x$.doi.org/10.21786/bbrc/14.9.17 to its volatile, nonlinear and non-parametric approach it is hard to predict right stock prices at right timing to buy or sell. Many researchers used various techniques for technical analysis and were not produced statistical valid result because highly subjective in nature of TA. In era of Artificial Intelligence, Data Mining techniques and Deep Learning techniques are taken into consideration for predict the direction of trends in stock market.

A. Sentiment Analysis: What other people think of me is not my Business" has always been makes us curious while indulge in decision making process. Earlier, many of us asked our personal acquaintances to recommend a broker to decide in which share we should invest our money. With the invent of Internet and world wide web have now let it happen to find out large pool of online users to share recommendation, opinion, views about an event. Although these online users are never our personal acquaintance nor professional critics. An irony of situation is many people are sharing opinions available to completely unknown person via Internet. In the beginning of 21st century, social networking websites taken exponential growth in popularity among online users by providing them a platform for discussion and exchange information. Apart from posting photos and videos for event, now online users are posting their comments, views, opinion about event. With this platform, most researchers are analyzing posts or opinion, performing computational methods to come to conclusion

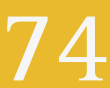


about popularity of event. This analysis part is becoming more powerful day by day to gauge user's opinion and would play a significant role for someone to make major decision in future.

With the rapid development of Information and Communication Technologies (ICT), social networking sites now have potential to transform the whole political arena into computerized world by introducing the whole new concept of generate, regenerate and share information in a wide scale of political context. The social microblogging sites such as Pinterest, Tumblr, Apsense, Scoop and specially Twitter are leading pools of online users and contains massive information that can be useful by extracting knowledge from information and that knowledge become asset for business specialist, political specialist, marketing specialist etc. In order to extract knowledge from information, Sentiment Analysis techniques can be used. Opinions are central to almost all human activities and influencers of our behaviors. Humans belief, perceptions of reality, choices that we make generally depends upon how others are evaluating things. For this reason, we often need to seek others opinions when we are in decision making process. This is fundamental truth for individual and for any organization as well. Sentiment Analysis also consider as Opinion Mining is a field of study that analyze people's emotions in most of the cases and a special field in text mining. It is a study that analyze people's emotions, views, opinion, attitudes towards entities such as product, individual, event, issue and their attribute.

B. Deep Learning: As subpart of Artificial Intelligence, Machine Learning presents a system that automatically train from concepts and knowledge with minimum programmed strategy. Generally, Machine Learning starts with collection of data, cleansing the data, feature generation and pattern recognition in the data for producing better prediction results. Deep Learning, a subpart of Machine Learning, principally works on Artificial Neural Network(ANN) where massive amount of data analyze with multivariate vectors. Deep Learning model typically relies on machine learning's algorithms where high level of data abstraction with multiple non-linear transformation taken into consideration for modelling.

In Sentiment Analysis, Deep Learning converts text data into dense numerical vectors before being initialized as the input layer of deep learning. With this, large number hidden layers improve the ability of feature extraction and transformation of deep learning. Many Deep Learning algorithms are explored by many researchers since 2011 from where it has been primarily observed two factors. One is the impact and correlation from a stock's price of company on/with other companies and second is growth rate of a company. Regression Neural Model and Recurrent Neural Network could be use respectively in mentioned factors to predict stock movement.

With the collection of Big Data from Web, Graphical Processing Unit(GPU) ability to parallel processing and presence of Convolutional Neural Network(CNN),
Deep Learning showed a wide presence in area of many computational applications including Object Detection, Image classification and time series predictions. Our primary aim of this paper to explored deep learning methods have been applied in prediction of stock movement in future. Although it would be beyond the scope of this paper to explore all factors require to perform prediction because of multivariate data. However, finding from various researchers presented in this paper could be insightful for other multivariate problems.

II. Literature Review: In this, authors give close eye on summarization, enhancements, challenges, future scope and categorized research papers published in this field since 2019. Research papers are categorized upon various parameters such as objective, algorithm, data scope, data set, their performance parameters and future scope. In Table: 1 about 25 papers inspected in this literature review organized in seven columns. Carta et. al., proposed to multiple agents in Deep RLL model for prediction of stock movement and stock prices. In this model, every agent was trained with diverse repetitions in intraday trading strategy rather Buy-Hold trading strategy. Agents were strictly trained on to perform exactly one course of action in trading period. Agents were trained either buy a stock and sell it before closing of a market or vice versa or trained not to perform any transaction in a whole day.

Authors created new data sets from original data sets on the basis of first open either week or day, last close of either week or a day and maximum days in either week or day. Further, authors applied multi resolution approach in time series (last 40 hours, last 20 days, last 8 weeks). Experiment performed on two sets of SEP500-S and S\&P500-L where S means (Short period: 2 Years) and L Means (long Period: 10 Years). Authors used walk-forward validation technique in order to validate time series data instead of cross validation approach like k-fold technique or leave-one-out from data which was sampled in random format on diverse folds. With this way training, test and validation considered from randomly fragmented folded data.

In this paper experimented the performance of ML models (RF and SVR) and DL models (DMLP, LSTM and CNN) in the stocks preselection before portfolio formation. For same, authors used data set of 9 years where 60 days daily returns for each stock as input feature to predict next day's return. The experiment was implemented by sliding window such as first four years for training data followed by one year as validation data followed by four years for testing data. For evaluation, authors used MAE, MSE as state of art parameters and observed that RF model outperformed SVR, DMLP, LSTM and CNN in stock prediction. In second phase of evaluation, authors claimed that RF+MVF and RF+OF performed best among models after deducted transaction fee (Caused by high turnover in real stock investment), based upon six metrics state of art parameters.

In paper published by Ingle et. al., Authors collected 
online news data from Yahoo Finance, Google News and Google Finance and then text mining was performed with common phenomenon of correlation between terms which further aggregated to text corpus. For text analysis, authors transformed raw text data into meaningful information by converting text to lower case, removed numbers and punctuation, removed stop words, stemming and identified synonym. From corpus, authors extracted TF-IDF features to identify companied used for stock prediction and their respective documents (news streams from web sources) were collected to prepare dataset. The models GLM, GBM and DL were used to predict stock movement and were evaluated with state of art parameters.

In this paper Althelaya et. al. adopted combination of multiresolution analysis such as SWT and EWT and deep learning architecture for data decomposition and data modelling/training respectively. For data decomposition, model adopted multi-scale wavelet analysis of financial time series data and set of deep learning networks were trained for each scale to performs prediction at each level. Further, multiple intermediate networks based upon number of resolutions layers generated from previous stage. For this, authors constructed each network using two layers of LSTM and one dense layer to learn each sub layer. Ahead of this, model consider input feature from forecast produced by the second stage along with construction LSTM layers and dense layer to produce final output using a linear activation function. Further, results of Empirical Multi- resolution wavelet analysis were compared with SWT's results.

In this paper, lee et. al., researchers collected dataset in order of 4000 daily time steps from three stock exchanges such as SEP 500, KOSPI200 and FTSE100 and dataset ratios were set to $0.7,0.1$ and 0.2 respectively for experiment the training, validation and test. Model was designed in view of understand the sequential pattern of target index and market components. For same, model was developed in two phases. In first phase, common features were extracted by input the data into series of two CNN layers and max-pool layer extracted core feature of company stock by applied max-operation between two companies at the time to sliding window and last four layers of ConvLSTM were learnt the spatiotemporal features of company's market size. In second phase, target index consisted of two stacks of LSTM in order to learn temporal pattern.

In this paper, Gupta et. al. introduced" Convolutional Transform Learning", learns a set of filters operated on observed sample to generate set of features Researchers choose NSE dataset contains information of 150 symbols between 2014 to 2018 and these stocks were chosen after filtering out stocks that had less than 3 years of data. Five raw inputs (Open price, Close price, High, Low, Net Asset Value) were considered in order to either buy stock or sell stocks keeping in mind the expectation of stock price on later day. Five raw inputs processed unsupervised by separate 1-D processing line and then concatenated and fed into Transform Learning Layer for fusion which led to supervised benchmark techniques with an output node (buy/ sell) for classification and real valued for regression. Lastly, authors compare it with state of art time series models such as TimeNet and ConvTimeNet. Paper published by Kumar et. al. proposed an intraday trading framework consisted three computational phases.

In first phase of model, researchers prepared dataset and for the same ninety days recent intraday trading data extracted from google finance about eighteen companies from six stock exchange. Later in this phase, researchers transformed whole data set into series of attributes such as open price, close price, trade volume, minimum price, maximum price etc. For this, researchers went one step ahead by adapted ten minutes' time resolution interval data from one-minute interval data for understand the market movement and stock momentum. In second phase, researchers used recursive feature engineering with logistic regression (RFE-LRC) in order to eliminate the noisy features and explore the optimum combination of features to avoid non-linearity correlation among them. And in last phase of framework, researchers explored series of hyper parameters that were put high impact on development of model. These hyper parameters were time- step, unseen layers, unseen neurons, size of batch and epochs. In paper published by Naik et. al.,Authors proposed a method to identify a trend in data using a combination of candlestick data and technical indicators. For Technical Indicators, authors took data from National Stock Exchange(NSE) and applied ten indicators and calculate based upon respective formulas. For Candlestick patterns, authors took data from NSE, extracted day low-high- open-close price, apply Japanese candlestick pattern on daily basis in order to generate output candlestick pattern.

Paper published by kim et. al researchers designed a stock chart CNN images by designing four stock images such as candlestick chart, line chart, bar chart and F-line chart using SPY (SEP 500) dataset and then analysis which chart image performed better. It had been noticed that bar chart image considered acceptable graph because it was re-designed using high volume trading data. After selection of bar chart image, author fused fundamental tool of stock such as volume information of a stock and performed experiment. Further, time series data and financial data were used in context to apply DL technology. Earlier, researchers used RNN in order to evaluate the sequential pattern in data but didn't work because the absence of gradient. Later, researchers used optimal LSTM-CNN model by merging of stock chart CNN and stock time LSTM using identical characteristics of stock chart image data and stock time series data. From dataset of 68k training data points, researchers considered $10 \mathrm{k}$ validation data points and 19k testing data points. With this, researchers predict the stock price for next five minutes by opening trading window for thirty minutes in roll of one minute. Oncharoen et. al. performed the study using the news headlines and historical price data described in Section 
IV. Data are split into three parts comprising training data, validation data, and test data. Researchers train the models using training data and then select the final models based on maximum accuracy of the validation data. Finally, the performance of each model is evaluated using the test data. Sezer et. al. obtained daily stock prices of Dow 30 stocks and daily Exchange Traded Funds(ETF) dataset from" Yahoo Finance" for training and testing purposes. Further, adapted sliding window with re-trained a model where 5 years were chosen for training followed by 1 year for testing. Extracted data labelled manually by determined the top and bottom points in sliding window. For image creation, technical indicators were considered to generate 15 X 15 image in different time intervals for each stock in a five years of period. In CNN analysis phase, nine layers were used with $3 \mathrm{X} 3$ filter size and then buy-sell decisions are made according to the predicted Buy, Sell, Hold labels.

In this paper Chung et. al. extracted data from" Bloomberg" for Korea Stock Price Index in time duration of 4203 trading days where each sample contained information on informative attributes (High, Low, Volume, Open and Close) out of which 80\% data used for training and 20\% data for holdout. Feature Selection: Five technical indicators and five historical values were employed as input variables (scaled into range of $[0,1]$ ), obtaining multidimensional time series and output of prediction model was the closing price the next day. Authors applied GA to investigate the optimal architectural factors, including the size of the time window to be fed a LSTM network, and derives results through this genetic search. Further, applied input embedding of the last 10 time steps, and optimized the architecture to verify the effectiveness of GA-LSTM model on holdout data. Paper published by Fischer et. al. where researchers performed their study in five stages.

In first stage, researchers collected data from Thomas Reuters about SEP500 stock index from 1989 to 2015 and prepared binary matrix in order to identify whether stock is an index or not. This way whole raw data split into training, test and validation data. In second stage, researchers used LSTM network where authors calculated each stock's one day returns and pile into feature vector and followed Binary classification for target. Further, authors applied LSTM network to recognize information which needs to be removed from previous stage of a cell and decides what information must have added into cell on based upon results of previous two stages. Authors evaluated their model's performance on basis of Mean of returns in a day, Standard Deviation of returns, Sharpe Ratio and precision. On basis of these, authors claimed their model outperformed memory-free classification methods.

In this paper Tsantekidis et. al., authors generated input data of 10 orders for each side of LOB which further describe by two values; Volume and Price. Since Price and Volume range is much greater that neural network's activation function so authors normalized data set with z-score (Mean Value and Standard Deviation) before fed into network. In order to predict the direction of stock price, authors generated mean between best bid price and best ask price which predicted stock upward or downward movement and used as target for classification model. In order to forecast mid-price movement of a stock, CNN was used and for training a model authors used batch of 16 samples where each sample consisted a sequence of 100 consecutive depths and further each depth consisted 40 values. To measure the performance of model, authors reported the mean recall, precision and F1 score and compared these with Linear SVM model, MLP model and Leaky Rectifiers.

Wenf et. al. prepared four set of data from online resources such as (a)NASDAQ and DJIA Indices (Open, Close, Price, Volume/E ratio, DoJ index) (b) Technical Indicators (Stochastic 0scillator-\%k, William-\%R, Relative Strength Index) of stock (c) Google News on the stock (Number of visited users on Wikipedia about stock. Further, authors performed Features Generations process where firstly authors integrated generated features with original data followed by generation of target matrix based on the movement of stock market and considered both as input to the system. In continuation to above, authors identified important features via visualization, data mining and Consolidated Features. Authors applied Decision Tree, SVM and ANN to predict stock movement and compared their results using 10-fold cross validation.

In this paper Patel et. al. used two stage fusion approach involves SVR in first and in second stage involves ANN, RF, and SVR resulting into SVR-ANN, SVR-RF and SVR-SVR prediction models. In two stage approach, authors employed SVR in first stage which describe the day along with input parameters (open, close, high, low, volume) and then to prepare outputs which consists ten technical indicators which further served as the inputs to the prediction models in the second stage. In prediction models, identify mapping transformation from technical parameters describing $(t+n)$ th day to $(t+n)$ th's day closing price. Authors formed 20\% tuning data of whole dataset to decide best combination of parameter values for each SVR in the first stage, which further divided into training data (80\%) and testing data $(20 \%)$ in order to minimize error in statistical parameters in second stage of model.

Paper published by Yoshihara et. al. where authors grouped news articles in routine where each news article is represented by standard bag -of-words model considered to be a word vectors used as input to model. Word Vector (0 or 1 ) represented uptrend and downtrend respectively, considered to be model's output. For experiment, authors collected dataset of ten prominent Nikkei companies from Nikkei newspaper from where news articles published in eight years considered to be a training data followed by news articles published in follow year as validation data and followed by news articles published in a follow year as test data. In order to transform articles into vectors, authors determined the vocabulary in line with morphological analysis 
and statistical tests. For computational time, authors fixed number of experiments to 5000 and computed chi-square scores in three possible directions such as upward, downward and neutral. Model was trained on RNN-RBM+DBN and its parameters were tuned on the validation data.

\section{DISCUSSION AND ANALYSIS}

Stock market prediction is dynamic research field and due to its volatile nature, it is hard to predict acute results in future. However, many researchers used enormous algorithms to predict high returns stock in intraday trading or buy and hold trading system, we analysed

\begin{tabular}{|c|c|c|}
\hline WoS Categories & Number of relevant article & \% Total Publications \\
\hline Computer Science Artificial Intelligence & 76 & $33.188 \%$ \\
\hline Engineering Electrical Electronic & 54 & $23.581 \%$ \\
\hline Computer Science Information Systems & 47 & 20.524 \\
\hline Computer Science Theory Methods & 27 & $11.790 \%$ \\
\hline Operations Research Management Science & 26 & $11.354 \%$ \\
\hline Telecommunications & 23 & $10.044 \%$ \\
\hline Computer Science Interdisciplinary Applications & 21 & $9.170 \%$ \\
\hline Economics & 21 & $9.170 \%$ \\
\hline Mathematics Interdisciplinary Applications & 19 & $8.297 \%$ \\
\hline Business Finance & 15 & $6.550 \%$ \\
\hline Engineering Multidisciplinary & 13 & $5.677 \%$ \\
\hline Multidisciplinary Sciences & 12 & $5.240 \%$ \\
\hline Computer Science Software Engineering & 9 & $3.930 \%$ \\
\hline Management & 9 & $3.930 \%$ \\
\hline Physics Applied & 8 & $3.493 \%$ \\
\hline Physics Multidisciplinary & 8 & $3.493 \%$ \\
\hline Social Sciences Mathematical Methods & 8 & $3.493 \%$ \\
\hline
\end{tabular}

Table 2. Wos Research Publications In Various Journals Since 2011 On Deep Learning With Stock Market

\begin{tabular}{|c|c|c|}
\hline Source titles & Record Count & $\%$ of 369 \\
\hline Expert Systems With Applications & 23 & $6.23 \%$ \\
\hline IEEE Access & 21 & $5.69 \%$ \\
\hline Neural Computing Applications & 9 & $2.43 \%$ \\
\hline Advances in Intelligent Systems and Computing & 8 & $2.16 \%$ \\
\hline IEEE International Joint Conference on Neural Networks IJCNN & 8 & $2.16 \%$ \\
\hline Lecture Notes in Artificial Intelligence & 8 & $2.16 \%$ \\
\hline Applied Sciences Basel & 7 & $1.89 \%$ \\
\hline IEEE international conference on big data & 7 & $1.89 \%$ \\
\hline Complexity & 6 & $1.62 \%$ \\
\hline Soft Computing & 6 & $1.62 \%$ \\
\hline
\end{tabular}


recent trends in research areas of stock market prediction models and found some challenges that can be research areas in coming days:

The Dataset: Although, every country in a globe has

Figure 1: Articles published in WoS category since 2011 under deep learning

\section{WOS CATEGORIES}

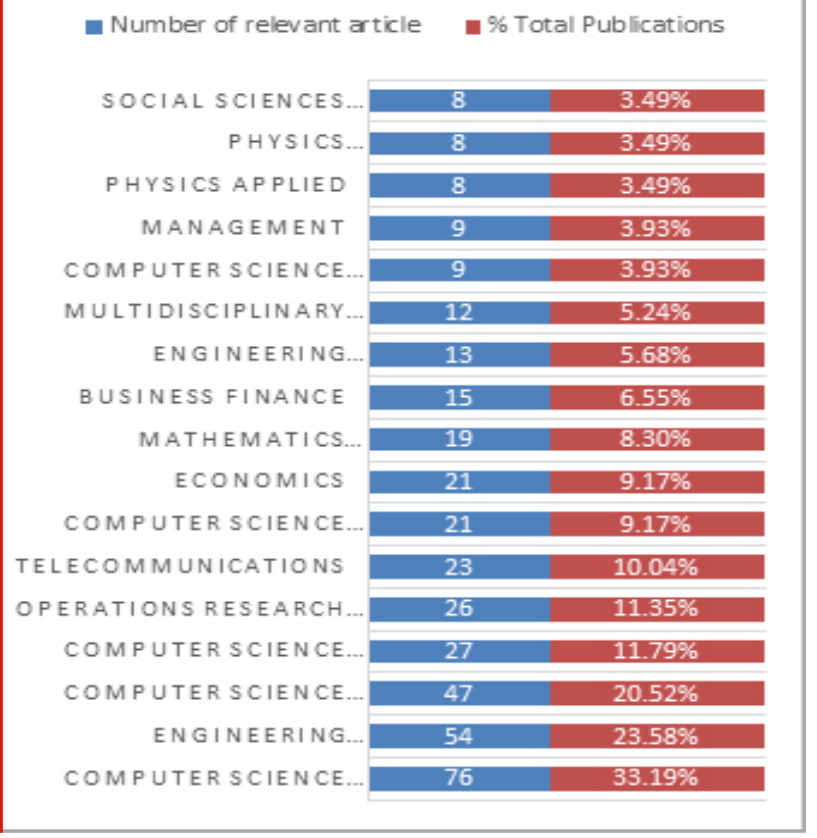

establish their respective exchange indexes with millions of transactions in a day. It is quite difficult to analyze whole dataset because of its wide range of technical indicators and fundamental strategies. Apart from technical indicators, one must take care of fundamental analysis of stocks before build a model as FA may not be a part of dataset. High Computational Power: Deep learning has capacity to solve complex problems due to its high level of abstract and presence of multiple layers. DL algorithms are design to inculcate routine input data of stock exchange and that lead to high-capacity GPU, storage and computational power.

Presence of Social media: Stock market highly volatile not only because of political or geographical change in society but also with some news from social media and news media. Sentiment analysis must play a significant role in prediction of stock movement to classifies the polarity in sentiment of news.

\section{CONCLUSION}

Stock market is diverse area of investment where one can invest across multiple entities such as intraday trading, buy and hold trading, commodity, power exchange etc., by pooling with number of small investments. As stock market data is in high volume, Deep learning is extremely successful in this area of research and if we follow its trend of success rates in other areas of research, definitely the use of its application genuinely takes us towards the acceptance. Stock market prediction with deep learning offers a platform for the common man where one can predict the results with some knowledge of stocks theory.

\section{REFERENCES}

A. Sharma, D. Bhuriya, and U. Singh, (2017) "Survey of stock market predic- tion using machine learning approach," Proceedings of the International Conference on Electronics, Communication and Aerospace Technology, ICECA 2017, vol. 2017-Janua, pp. 506509.

A. Tsantekidis, N. Passalis, A. Tefas, J. Kanniainen, M. Gabbouj, and A. Iosifidis,( 2017) "Forecasting stock prices from the limit order book using convolutional neural networks," Proceedings - 2017 IEEE 19th Conference on Business Informatics, CBI 2017, vol. 1, pp. 7-12.

A. W. Li and G. S. Bastos, (2020) "Stock Market Forecasting Using Deep Learning and Technical Analysis: A Systematic Review," IEEE Access, vol. 8, pp. 185 232-185 242.

A. Yoshihara, K. Fujikawa, K. Seki, and K. Uehara,( 2014) "Predicting stock market trends by recurrent deep neural networks," Lecture Notes in Com- puter Science (including subseries Lecture Notes in Artificial Intelligence and Lecture Notes in Bioinformatics), vol. 8862, pp. 759-769.

B. Weng, M. A. Ahmed, and F. M. Megahed,( 2017) "Stock market one-day ahead movement prediction using disparate data sources," Expert Systems with Applications, vol. 79, pp. 153-163. [Online]. Available: http://dx.doi.org/10.1016/j.eswa.2017.02.041

Enhanced Prediction of Intra-day Stock Market Using Metaheuristic Optimization on RNN-LSTM Network. pdf.

H. Chung and K. S. Shin, (2018) "Genetic algorithmoptimized long short-term memory network for stock market prediction," Sustainability (Switzer- land), vol. 10, no. 10.

J. Patel, S. Shah, P. Thakkar, and K. Kotecha, (2015) "Predicting stock market index using fusion of machine learning techniques," Expert Systems with Applications, vol. 42, no. 4, pp. 2162-2172. [Online]. Available: http:// dx.doi.org/10.1016/j.eswa.2014.10.031

K. A. Althelaya, S. A. Mohammed, and E. S. M. El-Alfy, (2021) "Combining deep learning and multiresolution analysis for stock market forecasting," IEEE Access, vol. 9, pp. 13 099-13 111.

Murphy and JJ, (1996) Technical Analysis of the Financial Markets: a Com- prehensive Guide to Trading Methods and Applications. New York Institute of Finance.

N. Naik and B. R. Mohan, (2019 ) "Intraday Stock Prediction Based on Deep Neural Network," National Academy Science Letters. [Online]. Available: https:// 
doi.org/10.1007/s40009-019-00859-1

0. B. Sezer and A. M. Ozbayoglu, (2018) "Algorithmic financial trading with deep convolutional neural networks : Time series to image conversion approach," Applied Soft Computing Journal, vol. 70, pp. 525538. [Online]. Available: https://doi.org/10.1016/j. asoc.2018.04.024

P. Gupta, J. Maggu, A. Majumdar, E. (2020) Chouzenoux, and G. Chierchia, "DeConFuse : a deep convolutional transform-based unsupervised fusion framework," vol. 5.

P. Hussein, B. Abdullah, and I. Technology, "A Systematic Review on Opinion Mining and Sentiment Analysis in Social Media Zaher Salah Abdel-Rahman F . Al-Ghuwairi Aladdin Baarah Ahmad Aloqail Bar ' a Qadoumi Momen Alhayek Bushra Alhijawi."

P. M. Tsang, P. Kwok, S. O. Choy, R. Kwan, S. C. Ng, J. Mak, J. Tsang, K. Koong, and T. L. Wong,( 2007) "Design and implementation of NN5 for Hong Kong stock price forecasting," Engineering Applications of Artificial Intelligence, vol. 20, no. 4, pp. 453-461.

P. Oncharoen and P. Vateekul, (2018) "Deep Learning for Stock Market Prediction Using Event Embedding and Technical Indicators," ICAICTA 2018 - 5th International Conference on Advanced Informatics: Concepts Theory and Applications, pp. 19-24.

S. Carta, A. Ferreira, A. S. Podda, D. Reforgiato Recupero, and A. Sanna, (2021) "Multi-DQN: An ensemble of Deep Q-learning agents for stock market forecasting," Expert Systems with Applications, vol. 164, no. November 2019, p. 113820. [Online]. Available: https://doi. org/10.1016/j.eswa.2020.113820

S. J. Park, Y. S. Lim, S. Sams, S. M. Nam, and H. W. Park,
(2011 ) "Networked politics on cyworld: The text and sentiment of Korean political profiles," Social Science Computer Review, vol. 29, no. 3, pp. 288-299.

S. W. Lee and H. Y. Kim,( 2020) "Stock market forecasting with super-high dimensional time-series data using ConvLSTM, trend sampling, and specialized data augmentation," Expert Systems with Applications, vol. 161, p. 113704. [Online]. Available: https: //doi. org/10.1016/j.eswa.2020.113704

T. Fischer and C. Krauss, (2018) "Deep learning with long short-term memory networks for financial market predictions," European Journal of Operational Research, vol. 270, no. 2, pp. 654-669. [Online]. Available: https:// doi.org/10.1016/j.ejor.2017.11.054

T. Kim and H. Y. Kim, (2019) "Forecasting stock prices with a feature fusion LSTM-CNN model using different representations of the same data," PLoS ONE, vol. 14, no. 2, pp. 1-23.

Y. F. Wang, (2002 ) "Predicting stock price using fuzzy grey prediction system," Expert Systems with Applications, vol. 22, no. 1, pp. 33-38,.

Y. M. Wadghule and V. Sonawane, (2017 ) "Stock market prediction and forecasting techniques: a survey," International Journal of Engineering Sciences and Research Technology, vol. 6, no. 1, pp. 39-42. [Online]. Available: http://www.ijesrt.com/issuespdffile/Archive2017/ January-2017/4.pdf

Y. Ma, R. Han, and W. Wang,( 2020) "Portfolio optimization with return prediction using deep learning and machine learning," Expert Systems with Applications, vol. 165, no. September, p. 113973, 2021. [Online]. Available: https://doi.org/10.1016/j. eswa.2020.113973 